\title{
Digital Image Correlation on FIB Ring-Core Measurement on Residual Stress of Thin Films
}

\author{
Yun-Chia Chou ${ }^{1}$, Wen-Yen Lin ${ }^{1}$, Wen-Chieh Pan ${ }^{1}$, Ming-Tzer Lin ${ }^{1 *}$, and Terry Yuan-Fang Chen ${ }^{2}$ \\ ${ }^{1}$ Graduate Institute of Precision Engineering, National Chung Hsing University Taichung, Taiwan \\ ${ }^{2}$ Department of Mechanical Engineering, National Cheng Kung University, Tainan, Taiwan \\ *Corresponding author : mingtlin@nchu.edu.tw
}

\section{Extended Abstract}

The residual stress generated during the deposition of the film may cause unpredictable deformation or damage to the component; therefore, it is vital to understand residual stress. Reliable measurement and modelling of residual stresses at the micron-scale for small-scale structures and nanostructured materials is a tremendously challenging task. The analysis of depth profiles of residual strain requires detailed knowledge of in-depth lattice strain function in order to calculate the residual stress profile in a manner that considers both the mechanical anisotropy and the texture of the materials.

Full-field measurement of the deformation behaviour of small scale structures and nanostructured materials has recently been made possible by combining Scanning Electron Microscopy (SEM) and Digital Image Correlation (DIC) techniques. The DIC techniques were first introduced by Sutton who used the calculation of a grayscale digital image through bilinear interpolation for the object translation. It was suggested that a discrete digital image be interpolated for a continuous smooth secondary function. Although a high-quality pattern can be artificially applied for a better measurement, the mechanical behaviour of sample structures may become affected by the patterning process. The high magnification of SEM-DIC leads to complex image distortions. Long image scanning times can capture high-noise sensitivity and drift from SEM parameters; therefore, the development of a proper scheme for digital correlation of SEM images with natural surface features is needed.

Prior to this present research, a study was made on the methodology and validation of DIC under SEM to minimize errors and use it for surface deformation and strain measurement of CMOS IC samples with multiple thin layers, and to validate the DIC method and its parameters using SEM images under spatial translation or time. For the present study, DIC with proper image correlation criteria postulated in the previous study was performed to obtain stability and accuracy for the specimen images. The images were acquired by incremental focused ion beam (FIB) ring-core drilling with various depth steps. The FIB-DIC was then used to calculate the residual stress. This study used a semi-destructive measurement method, which is not limited to crystalline and amorphous materials, and is suitable for analyzing uneven structures. Further, several semidestructive measurement methods were used to gauge the strain relief when the material was removed or deformed.

Reliable measurement and modeling of residual stresses at the micrometer scale for small-scale structures and nanostructured thin films is a challenging task. The analysis of the depth profiles of the residual strain requires detailed knowledge of the in-depth lattice strain function, so the residual stress profile calculated in a manner that considers the mechanical anisotropy and texture of the materials. The development of a microstructure independent procedure for depth-resolved measurement of residual stress is an issue of strategic interest. In this study, a digital correlation (DIC) of the specimen images acquired by incremental focused ion beam (FIB) ring-core drilling with various depth steps was performed. A translation test was performed first to study the applicability of DIC to FIB images, and a proper procedure was established to obtain more accurate results. Moreover, state of the film in terms of the residual stress was evaluated and compared to illustrate the feasibility of this technique. 